www.nature.com/clinicalpractice/neuro

with PSP from controls and patients with parkinsonian syndromes or neurodegenerative illnesses that lead to dementia.

CSF samples from 21 patients with PSP, 118 patients with another neurodegenerative disorder (44 with frontotemporal dementia, 20 with corticobasal degeneration syndrome, 29 with Alzheimer disease, 10 with Parkinson disease and 15 with dementia and Lewy bodies) and 27 individuals without any neurodegenerative disorder were analyzed by semiquantitive immunoprecipitation.

The ratio of truncated tau to extended tau in patients with PSP was approximately half that in the other subgroups $(0.504 \pm 0.284$, vs mean range $0.899-1.215 ; P<0.005)$. The magnitude of the tau ratio in patients with PSP was inversely correlated with the severity of disease. Further analysis determined a cut-off ratio of 0.62 , below which approximately $85 \%$ of patients with PSP could be reliably diagnosed. In the subgroup of 44 patients with frontotemporal dementia who underwent MRI, those with a tau ratio below the cut-off value had significant clusters of reduced gray matter density in the brainstem.

The authors suggest that a low tau ratio could indicate altered tau processing and susceptibility to brainstem atrophy, as seen in patients with PSP.

Original article Borroni B et al. (2008) Tau forms in CSF as a reliable biomarker for progressive supranuclear palsy. Neurology 71: 1796-1803

\section{Once-daily extended-release levetiracetam reduces seizure frequency}

Increased numbers of daily antiepileptic drug doses are associated with reduced treatment compliance among patients with epilepsy, which might impair seizure control. Peltola et al. investigated the safety and efficacy of extended-release levetiracetam (LEV XR), which need only be administered once daily, as adjunctive therapy in patients with partial-onset seizures (POSs).

This multicenter, double-blind trial examined patients aged 12-70 years with recurrent POS refractory to between one and three antiepileptic drugs. Seizure frequency was monitored for 8 weeks to determine baseline POS frequency. Participants were then randomly allocated to receive either two $500 \mathrm{mg}$ tablets of LEV XR daily $(n=74)$ or two matching placebo tablets $(n=178)$ for 12 weeks, with concomitant antiepileptic treatment.

The median reduction from baseline in POS frequency was significantly greater in the LEV XR group than in the placebo group (46.07\% vs $33.40 \%$ reduction, respectively). In total, 34 (43\%) LEV XR patients and 23 (29\%) placebo patients experienced $a \geq 50 \%$ reduction from baseline in weekly POS frequency, and $8(10.1 \%)$ LEV XR patients and 1 (1.3\%) placebo patient, were POS-free for the whole 12-week treatment period. Treatment-emergent and therapy-related adverse effects were similar in the two study groups.

The authors conclude that once-daily LEV XR significantly reduces POS frequency and is well tolerated in patients with seizures refractory to at least one antiepileptic drug.

Original article Peltola J et al. (2008) Once-daily extendedrelease levetiracetam as adjunctive treatment of partialonset seizures in patients with epilepsy: a double-blind, randomized, placebo-controlled trial. Epilepsia [doi: 10.1111/ j.1528-1167.2008.01817.x]

\section{Flupirtine is a promising agent for the treatment of autoimmune optic neuritis}

Currently, no approved therapies are available to treat neuronal and axonal degeneration associated with chronic progressive multiple sclerosis. In a new study, Sättler et al. have reported that flupirtine, a nonopioid analgesic approved for long-term use in humans, has neuroprotective effects in a rat model of optic neuritis induced by myelin oligodendrocyte glycoprotein. In animal models, flupirtine has previously shown neuroprotective properties associated with upregulation of the antiapoptotic protein $\mathrm{Bcl}-2$. Downregulation of $\mathrm{Bcl}-2$ has been implicated in the degeneration of retinal ganglion cells in autoimmune optic neuritis.

In the current study, rats received one of four treatments: interferon- $\beta 1 \mathrm{a}$ monotherapy $(300,000 \mathrm{U}$ three times weekly), flupirtine monotherapy ( $\sim 2.45 \mathrm{mg} / \mathrm{kg}$ body weight daily), interferon- $\beta 1 \mathrm{a}$ and flupirtine combination therapy, or vehicle treatment (control group). Retinal ganglion cells (labeled at the start of the experiments) in rats treated with flupirtine 